\title{
Recommendations for Use of Meningococcal Conjugate Vaccines in HIV-Infected Persons - Advisory Committee on Immunization Practices, 2016
}

\author{
Jessica R. MacNeil, MPH${ }^{1}$; Lorry G. Rubin, MD²; Monica Patton, MD¹; Ismael R. Ortega-Sanchez, PhD³; Stacey W. Martin, MS
}

At its June 2016 meeting, the Advisory Committee on Immunization Practices (ACIP) recommended routine use of meningococcal conjugate vaccine (serogroups $\mathrm{A}, \mathrm{C}, \mathrm{W}$, and Y; including MenACWY-D [Menactra, Sanofi Pasteur] or MenACWY-CRM [Menveo, GlaxoSmithKline]) for persons aged $\geq 2$ months with human immunodeficiency virus (HIV) infection. ACIP has previously recommended routine vaccination of persons aged $\geq 2$ months who have certain medical conditions that increase risk for meningococcal disease (1), including persons who have persistent (e.g., genetic) deficiencies in the complement pathway (e.g., C3, properdin, Factor D, Factor H, or C5-C9); persons receiving eculizumab (Soliris, Alexion Pharmaceuticals) for treatment of atypical hemolytic uremic syndrome or paroxysmal nocturnal hemoglobinuria (because the drug binds $\mathrm{C} 5$ and inhibits the terminal complement pathway); and persons with functional or anatomic asplenia (including persons with sickle cell disease). Routine vaccination with meningococcal conjugate vaccine is also recommended for all healthy adolescents in the United States (1). This report summarizes the evidence considered by ACIP in recommending vaccination for HIV-infected persons, and provides recommendations and guidance for use of meningococcal conjugate vaccines (serogroups $\mathrm{A}, \mathrm{C}, \mathrm{W}$, and $\mathrm{Y}$ )

Recommendations for routine use of vaccines in children, adolescents, and adults are developed by the Advisory Committee on Immunization Practices (ACIP). ACIP is chartered as a federal advisory committee to provide expert external advice and guidance to the Director of the Centers for Disease Control and Prevention (CDC) on use of vaccines and related agents for the control of vaccine-preventable diseases in the civilian population of the United States. Recommendations for routine use of vaccines in children and adolescents are harmonized to the greatest extent possible with recommendations made by the American Academy of Pediatrics (AAP), the American Academy of Family Physicians (AAFP), and the American College of Obstetricians and Gynecologists (ACOG). Recommendations for routine use of vaccines in adults are harmonized with recommendations of $A A F P$, ACOG, and the American College of Physicians (ACP). ACIP recommendations approved by the CDC Director become agency guidelines on the date published in the Morbidity and Mortality Weekly Report (MMWR). Additional information is available at http://www.cdc.gov/vaccines/acip. among HIV-infected persons aged $\geq 2$ months; the majority of meningococcal disease among HIV-infected persons is caused by these four serogroups.

\section{Methods}

The ACIP Meningococcal Vaccines Work Group reviewed the immunogenicity and safety data from two studies of MenACWY-D in HIV-infected persons (2-4) during monthly teleconferences. No studies of immunogenicity or safety of MenACWY-CRM in HIV-infected persons are available. According to a nonsystematic literature search of PubMed using the search terms "meningococcal conjugate vaccine," "quadrivalent," and "HIV," and consultation with the manufacturers, these two studies represent all known evidence for the immunogenicity and safety of these vaccines in HIV-infected persons. The work group also evaluated the evidence and unpublished surveillance data regarding meningococcal disease epidemiology among HIV-infected persons in the United States and a cost-effectiveness analysis of routine vaccination of HIV-infected persons. The cost-effectiveness analysis of routine vaccination versus no vaccination of HIV-infected persons was conducted assuming an initial vaccine efficacy of $75 \%$ (range $=37 \%-91 \%$ ) for persons with a high CD4 count and $37 \%$ (range $=24 \%-60 \%$ ) for persons with a low CD4 count based on the immunogenicity data reported in the literature for MenACWY-D (2-4). A summary of the data reviewed and work group discussions was presented to ACIP; recommendations for use of meningococcal conjugate vaccines among HIV-infected persons aged $\geq 2$ months were approved by ACIP at its June 22, 2016 meeting (detailed meeting minutes are available at http://www.cdc.gov/vaccines/acip/ meetings/meetings-info.html).

CDC vaccine recommendations are developed using the Grading of Recommendations, Assessment, Development, and Evaluation (GRADE) framework (http://www.cdc.gov/ vaccines/acip/recs/grade/index.html). The type and quality of available evidence supporting the use of meningococcal conjugate vaccines among HIV-infected persons aged $\geq 2$ months were evaluated using GRADE. There is no available evidence for safety or effectiveness of these vaccines in HIV-infected persons aged $<2$ years or $\geq 25$ years. 


\section{Meningococcal Disease in HIV-Infected Persons}

Surveillance data for cases of meningococcal disease among HIV-infected persons in the United States are limited. The HIV status of patients with meningococcal disease is routinely captured in Active Bacterial Core surveillance (ABCs), an active population-based and laboratory-based surveillance system that operates in 10 sites, representing a population of approximately 43 million persons, or $14 \%$ of the U.S. population (5). However, the HIV status of patients with meningococcal disease is not reported through the National Notifiable Diseases Surveillance System (NNDSS), a passive surveillance system that operates in all U.S. states and territories. During 1995-2014, a total of 62 cases of meningococcal disease among HIV-infected persons were reported to ABCs; these cases represent 2\% of the 3,951 meningococcal disease cases reported to $\mathrm{ABC}$ s during that period (CDC, unpublished data, 2016). Thirteen (21\%) cases were serogroup B, 23 (37\%) were serogroup $C$, three (5\%) were serogroup W, 17 (27\%) were serogroup $\mathrm{Y}$, and six (10\%) were other/unknown serogroups (CDC, unpublished data, 2016). The majority (92\%) of cases of meningococcal disease among HIV-infected persons occurred in adults aged 20 through 59 years.

Although surveillance data for cases of meningococcal disease among HIV-infected persons are limited in the United States, a growing body of evidence demonstrates an increased risk for meningococcal disease among HIV-infected persons. In studies from South Africa, the United States, and the United Kingdom, the incidence of meningococcal disease in
HIV-infected persons ranged from 3.4 to 6.6 per 100,000 (relative risk $=5-13$ compared with HIV-uninfected persons) (Table 1) (6-9). Among HIV-infected persons, a low CD4 count or high viral load were associated with an increased risk (7). Similar increased risk for meningococcal disease was observed for both males and females with HIV infection $(7,9)$.

Data on the case-fatality ratio of meningococcal disease in HIV-infected persons vary: in the South Africa study, the casefatality ratio among HIV-infected patients was 20\%, compared with $11 \%$ among patients who did not have HIV infection (G). However, in the most recent studies from New York City and the United Kingdom, the meningococcal disease case fatality ratio observed among HIV-infected patients was lower than that among HIV-uninfected patients $(7,8)$.

\section{MenACWY-D Immunogenicity and Safety in HIV-Infected Persons}

The immunogenicity and safety of MenACWY-D in 324 HIV-infected adolescents and young adults aged 11 through 24 years were evaluated in an open-label trial with a randomized second dose component $(2,4)$. At study entry, participants received 1 dose of MenACWY-D. At 24 weeks, participants with CD4 percentage (the percentage of total lymphocytes that are CD4 cells) (CD4\%) $\geq 15 \%$ were randomized to receive or not receive a second dose of MenACWY-D; all participants with $\mathrm{CD} 4 \%<15 \%$ received a second dose. Vaccine effectiveness was inferred from serum bactericidal antibodies, measured using a serum bactericidal assay with a rabbit complement

TABLE 1. Evidence of increased risk for meningococcal disease among HIV-infected persons compared with HIV-uninfected persons — seven study populations, 1996-2013

\begin{tabular}{|c|c|c|c|c|c|}
\hline Period & Study site & Age group & No. of cases* & $\begin{array}{l}\text { Increase in meningococcal disease rate } \\
\text { among HIV-infected compared with } \\
\text { HIV-uninfected persons }\end{array}$ & Serogroups \\
\hline 1996-1999 & Australia ${ }^{\dagger}$ & All ages & 60 & 5-fold & $\mathrm{B}, \mathrm{C}$ \\
\hline 1990-2000 & London $\S$ & All ages & 2,900 & 14-fold & $\mathrm{B}, \mathrm{C}$ \\
\hline 1988-1993 & Atlanta, Georgia? & $18-45$ years & 132 & 24-fold & $B, C, Y$ \\
\hline $2003-2007$ & South Africa** & All ages & 504 & 11-fold & $A, B, C, W, Y$ \\
\hline 2000-2008 & United States $\mathrm{ABCs} \mathrm{s}^{\dagger \dagger}$ & 25-64 years & 491 & 13-fold & $B, C, W, Y$ \\
\hline 2000-2011 & New York City ${ }^{\S}$ & $15-64$ years & 265 & 10-fold & $C, Y$ \\
\hline 2011-2013 & United Kingdom ๆๆ & All ages & 2,353 & 5 -fold & $A, B, C, W, Y$ \\
\hline
\end{tabular}

Abbreviations: $A B C s=$ Active Bacterial Core surveillance; HIV = human immunodeficiency virus.

* Total number of meningococcal disease cases reported during the study period regardless of HIV infection status

† Couldwell DL. Invasive meningococcal disease and HIV coinfection. Commun Dis Intell Q Rep 2001;25:279-80.

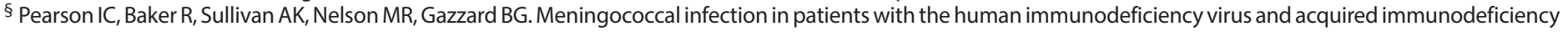
syndrome. Int J STD AIDS 2001;12:410-1.

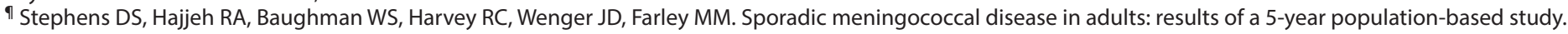
Ann Intern Med 1995;123:937-40.

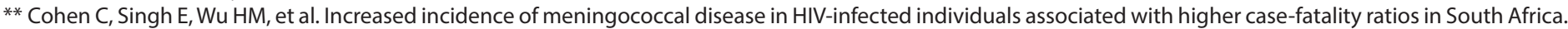
AIDS 2010;24:1351-60.

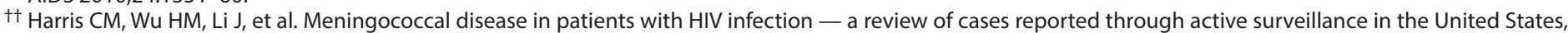
2000-2008. Open Forum Infect Dis 2016. Epub October 24, 2016. http://ofid.oxfordjournals.org/content/early/2016/10/24/ofid.ofw226.full.pdf+html.

$\S \S$ Miller L, Arakaki L, Ramautar A, et al. Elevated risk for invasive meningococcal disease among persons with HIV. Ann Intern Med 2014;160:30-7.

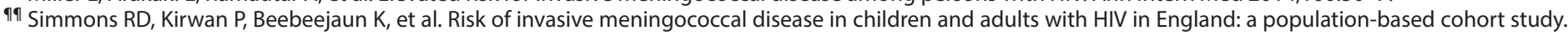
BMC Med 2015;13:297. 
source (rSBA). Rates of seroresponse (proportion of subjects with $\mathrm{a} \geq$ fourfold rise in $\mathrm{rSBA}$ titer compared with the baseline titer) against each meningococcal serogroup (A, C, W, Y), geometric mean titers (GMT), and the percentage of subjects with $\mathrm{rSBA}$ at or above a predefined titer $(\geq 1: 128)$ were determined from sera obtained at study entry and at weeks 4, 24, 28, and 72. Adverse events (AEs) were assessed for 6 weeks after each MenACWY-D dose through active follow-up $(2,4)$.

Among participants with CD $4 \% \geq 15 \%$ who received 1 vaccine dose, the proportions of participants with $\mathrm{rSBA}$ titers $\geq 1: 128$ at 4,28 , and 72 weeks were $65 \%, 31 \%$, and $21 \%$, respectively, against serogroup C, and $83 \%, 75 \%, 63 \%$, respectively, against serogroup Y (2). Among participants with $\mathrm{CD} 4 \% \geq 15 \%$ who received 2 doses (at 0,24 weeks), the proportions of participants with rSBA titers $\geq 1: 128$ at 4 , 28, and 72 weeks were $59 \%, 64 \%$, and $35 \%$, respectively, against serogroup C; and 73\%, 83\%, 71\%, respectively, against serogroup Y (2). Among participants with CD $4 \%<15 \%$, all of whom received 2 doses (at 0,24 weeks), the proportions of participants with rSBA titers $\geq 1: 128$ at 4,28 , and 72 weeks were $22 \%, 22 \%$, and $6 \%$, respectively, against serogroup C, and $30 \%, 30 \%, 28 \%$, respectively, against serogroup Y (2). A serious AE was experienced by $2.2 \%-6.5 \%$ of participants through 6 weeks post-vaccination*; one serious AE (ocular pain) was judged to be related to MenACWY-D. Serious AE rates were inversely related to entry $\mathrm{CD} 4 \%$. Two deaths were reported, but both were determined to be unrelated to the vaccine $(2,4)$.

The immunogenicity and safety of MenACWY-D in 59 HIV-infected children aged 2 through 10 years with CD4\% $\geq 25 \%$ was evaluated in an open label trial (3). Participants received MenACWY-D at study entry and at week 24 . Vaccine effectiveness was inferred from serum bactericidal antibodies, measured using a serum bactericidal assay with rSBA. Rates of seroresponse (proportion of subjects with a $\geq$ fourfold rise in post-vaccination $\mathrm{rSBA}$ titer compared with the baseline titer) against each meningococcal serogroup (A, C, W, Y), GMTs, and the percentage of subjects with rSBA at or above a predefined titer $(\geq 1: 128)$ were determined from sera obtained at entry and weeks 4, 24, 28, and 72. Study participants were assessed for AEs 6 weeks after each MenACWY-D dose (3).

The proportion of participants with $\mathrm{rSBA}$ titers $\geq 1: 128$ after 1 dose (week 4) and 2 doses (week 28) of MenACWY-D were $96 \%$ and $96 \%$, respectively, for serogroup A, $49 \%$ and $80 \%$, respectively, for serogroup C, $98 \%$ and $100 \%$, respectively, for serogroup $\mathrm{W}$, and $90 \%$ and $98 \%$, respectively, for serogroup Y (3). At week 72 the proportions of participants

\footnotetext{
* Serious adverse events (AEs) defined as Guillain-Barré syndrome, death, and new grade 3 or higher AE according to the December 2004 Division of AIDS AE Grading Table.
}

with rSBA titers $\geq 1: 128$ were $80 \%$ for serogroup A, $45 \%$ for serogroup C, $95 \%$ for serogroup W, and $91 \%$ for serogroup $Y$ (3). Overall, $5 \%$ of participants reported a serious AE; no AE was judged to be related to MenACWY-D (3).

Evidence supporting the use of meningococcal conjugate vaccines in HIV-infected persons was evaluated using the GRADE framework and was determined to be type 3 (low level of evidence) (Table 2). The recommendation was designated as Category A (recommended for all persons in an age-based or risk factor-based group) because of the epidemiologic data supporting an increase in risk for meningococcal disease among HIV-infected persons.

From a lifetime perspective, it is estimated that, compared with no vaccination, approximately 122 (95\% confidence interval $[\mathrm{CI}]=116-129)$ cases and $23(\mathrm{CI}=18-29)$ deaths could be prevented, and $385(\mathrm{CI}=230-458)$ quality-adjusted life years (QALYs) could be saved, at a mean cost per QALY of $\$ 732,000(\mathrm{CI}=\$ 337,000-\$ 1,218,000)$ with a meningococcal conjugate vaccination program that includes a primary vaccination series followed by lifelong booster doses until age 70 years, targeting all currently HIV-infected persons aged $\geq 2$ months in the United States (CDC, unpublished data, 2016). ${ }^{\dagger}$

\section{Recommendations}

HIV-infected persons aged $\geq 2$ months should routinely receive meningococcal conjugate vaccine (Table 3 ). HIVinfected children aged $<2$ years should receive the vaccine in accordance with the age-appropriate, licensed, multidose schedule $(1,10)$. Persons aged $\geq 2$ years with HIV infection who have not been previously vaccinated should receive a 2-dose primary series of MenACWY conjugate vaccine. Persons aged $\geq 2$ years with HIV infection who have been previously

\footnotetext{
\rceil Unpublished data, ACIP meeting June 2016. Key model assumptions were presented at the June 2016 ACIP meeting. Methods described in Shepard CW, Ortega-Sanchez IR, Scott RD 2nd, Rosenstein NE. Cost-effectiveness of conjugate meningococcal vaccination strategies in the United States. Pediatrics 2005; 115:1220-32.
}

TABLE 2. Summary of evidence for meningococcal conjugate vaccination of HIV-infected persons aged $\geq 2$ months using the Grading of Recommendations, Assessment, Development, and Evaluation (GRADE)* framework - United States

\begin{tabular}{lc}
\hline Outcome & $\begin{array}{c}\text { Evidence } \\
\text { type }^{\dagger}\end{array}$ \\
\hline Benefits & 3 \\
Short-term immunogenicity 4 weeks after 1 dose (week 4) & 3 \\
Short-term immunogenicity 4 weeks after 2 doses (week 28) & 3 \\
Persistence of immunogenicity 48 weeks after 2 doses (week 72) & 3 \\
Harms & 4 \\
\hline Serious adverse events (after any dose) & \\
\hline
\end{tabular}

* http://www.cdc.gov/vaccines/acip/recs/grade/index.html.

† Evidence type: 1 = highest level of evidence; 2 = high level of evidence; $3=$ low level of evidence; 4 = lowest level of evidence. 
Morbidity and Mortality Weekly Report

TABLE 3. Recommended meningococcal conjugate vaccination schedule and intervals for HIV-infected persons - Advisory Committee on Immunization Practices, United States, 2016

\begin{tabular}{|c|c|}
\hline Age group & Recommended schedule and intervals \\
\hline \multicolumn{2}{|l|}{ Primary vaccination } \\
\hline \multirow[t]{2}{*}{$<2$ years } & 4 doses of MenACWY-CRM (Menveo)* at ages $2,4,6$, and $12-15$ months \\
\hline & 2 doses of MenACWY-D (Menactra) at age 9-23 months, 12 weeks apart ${ }^{\dagger, \S, n}$ \\
\hline$\geq 2$ years & 2 doses of MenACWY-D or MenACWY-CRM, 8-12 weeks apart ${ }^{\dagger, 9}$ \\
\hline \multicolumn{2}{|l|}{ Booster dose } \\
\hline$<7$ years at previous dose & Additional dose of MenACWY-D or MenACWY-CRM 3 years after primary series; boosters should be repeated every 5 years thereafter** \\
\hline 77 years at previous dose & Additional dose of MenACWY-D or MenACWY-CRM 5 years after primary series; boosters should be repeated every 5 years thereafter \\
\hline \multirow{6}{*}{\multicolumn{2}{|c|}{$\begin{array}{l}\text { * MenACWY-CRM is licensed for use in persons aged } 2 \text { months through } 55 \text { years. Children aged } 7 \text { through } 23 \text { months who initiate vaccination with MenACWY-CRM } \\
\text { should receive } 2 \text { doses } 12 \text { weeks apart, with the second dose administered after the first birthday. Source: Food and Drug Administration. Menveo U.S. package } \\
\text { insert. http://www.fda.gov/downloads/BiologicsBloodVaccines/Vaccines/ApprovedProducts/UCM201349.pdf. } \\
+ \text { MenACWY-D is licensed for use in persons aged } 9 \text { months through } 55 \text { years. Source: Food and Drug Administration (FDA). Menactra U.S. package insert. http:// } \\
\text { www.fda.gov/downloads/BiologicBloodVaccines/Vaccines/ApprovedProducts/UCM131170.pdf. } \\
\S \text { If MenACWY-D is used, it should be administered at least } 4 \text { weeks after completion of all pneumococcal conjugate vaccine doses. } \\
\text { " If MenACWY-D is to be administered to a child at increased risk for meningococcal disease, including children with HIV infection, it is recommended that MenACWY-D } \\
\text { be given either before DTaP or concomitantly with DTaP. }\end{array}$}} \\
\hline & \\
\hline & \\
\hline & \\
\hline & \\
\hline & \\
\hline & \\
\hline
\end{tabular}

vaccinated with 1 dose of meningococcal conjugate vaccine should receive a booster dose at the earliest opportunity, provided at least 8 weeks have elapsed since the previous dose, and then continue to receive boosters at the appropriate interval throughout life. ${ }^{\S}$ The recommendations for children aged 2 months through 2 years and persons aged $\geq 25$ years are based on expert opinion; the vaccine was not studied in HIV-infected persons in these age groups. On the basis of available data and expert opinion, either MenACWY-CRM or MenACWY-D may be used in HIV-infected persons.

The same vaccine product should be used for all doses. However, if the product used for previous doses is unknown or unavailable, the vaccination series may be completed with any age- and formulation-appropriate meningococcal conjugate vaccine. Although no data on interchangeability of meningococcal conjugate vaccines in HIV-infected persons are available, limited data from a postlicensure study in healthy adolescents suggests safety and immunogenicity of MenACWY-CRM are not adversely affected by prior immunization with MenACWY-D $(1,11)$.

ACIP recommends that HIV-infected infants aged 2 through 23 months receive MenACWY-CRM. HIV-infected children should not receive MenACWY-D before age 2 years, similar to the recommendation for children with functional or anatomic asplenia. Previously, children with functional or anatomic asplenia were recommended to receive 13-valent pneumococcal conjugate vaccine (PCV13) according to the normal schedule but to delay MenACWY-D vaccination until age 2 years because of immune interference $(1,12)$. Because MenACWY-CRM does not demonstrate immune interference

\footnotetext{
\$If the most recent dose was received before age 7 years, a booster dose should be administered 3 years later. If the most recent dose was received at age $\geq 7$ years, a booster dose should be administered 5 years later.
}

with 7-valent pneumococcal conjugate vaccine (PCV7) after the 12-month dose (13-15), MenACWY-CRM can be administered concomitantly with PCV13.

In addition, new data suggest the potential for immunologic interference in the meningococcal human complement serum bactericidal assay (hSBA) responses when MenACWY-D is administered 30 days after Daptacel (diphtheria and tetanus toxoids and acellular pertussis vaccine [DTaP], Sanofi Pasteur) (16). In one study among children aged 4 through 6 years, the hSBA responses to all four meningococcal serogroups failed to meet noninferiority criteria when MenACWY-D was administered 30 days after Daptacel. In contrast, co-administration of MenACWY-D and Daptacel was not associated with reduced hSBA responses to all four meningococcal serogroups. The study objectives did not include evaluation of the potential for interference that other DTaP containing vaccines might have on meningococcal seroresponse rates (16). If MenACWY-D is to be administered to a child at increased risk for meningococcal disease, including children who are HIV-infected, it is recommended that MenACWY-D be given either before or concomitantly with DTaP.

MenACWY is recommended for HIV-infected persons aged $\geq 56$ years because of the need for revaccination (i.e., booster doses). Meningococcal polysaccharide vaccine (MPSV4, Menomune, Sanofi Pasteur) is the only licensed meningococcal vaccine for adults aged $\geq 56$ years; however, no data are available on use of MPSV4 in HIV-infected adults. For healthy adults who have received MenACWY previously, limited data demonstrate a higher antibody response after a subsequent dose of MenACWY compared with a subsequent dose of MPSV4 (1).

To date, no randomized, controlled clinical trials have been conducted to evaluate use of MenACWY vaccines in pregnant or lactating women. Pregnancy should not preclude indicated vaccination with MenACWY. 


\section{Summary}

What is currently recommended?

The Advisory Committee on Immunization Practices (ACIP) currently recommends routine vaccination with meningococcal conjugate vaccine for all adolescents and for certain groups of persons at increased risk for meningococcal disease: persons who have persistent complement component deficiencies; persons who have anatomic or functional asplenia; microbiologists who routinely are exposed to isolates of Neisseria meningitidis; persons identified to be at increased risk because of a meningococcal disease outbreak attributable to serogroup $A, C$, $W$, or Y; military recruits; first-year college students living in residence halls; and persons who travel to or reside in areas in which meningococcal disease is hyperendemic or epidemic. In addition, ACIP recommends routine vaccination with serogroup B meningococcal (MenB) vaccine for persons who have persistent complement component deficiencies; persons who have anatomic or functional asplenia; microbiologists who routinely are exposed to isolates of $\mathrm{N}$. meningitidis; and persons identified to be at increased risk because of a serogroup $B$ meningococcal disease outbreak.

Why are the recommendations being modified now?

A growing body of evidence supports an increased risk for meningococcal disease in human immunodeficiency virus (HIV)-infected persons. The evidence supporting the use of meningococcal conjugate vaccines in HIV-infected persons was evaluated using the Grading of Recommendations, Assessment, Development, and Evaluation (GRADE) framework.

What are the new recommendations?

All HIV-infected persons aged $\geq 2$ months should routinely receive meningococcal conjugate vaccine; children aged $<2$ years should be vaccinated using a multidose schedule. Persons aged $\geq 2$ years with HIV who have not been previously vaccinated should receive a 2-dose primary series of meningococcal conjugate vaccine. Persons with HIV who have been previously vaccinated with meningococcal conjugate vaccine should receive a booster dose at the earliest opportunity (at least 8 weeks after the previous dose) and then continue to receive boosters at the appropriate intervals. If the most recent dose was received before age 7 years, a booster dose should be administered 3 years later. If the most recent dose was received at age $\geq 7$ years, a booster should be administered 5 years later and every 5 years thereafter throughout life.

\section{Precautions and Contraindications}

Before administering meningococcal conjugate vaccines, health care providers should consult the package insert for precautions, warnings, and contraindications $(13,16)$. Adverse events occurring after administration of any vaccine should be reported to the Vaccine Adverse Event Reporting System (VAERS). Reports can be submitted to VAERS online, by fax, or by mail. Additional information about VAERS is available by telephone (1-800-822-7967) or online (https://vaers.hhs.gov).

\section{Acknowledgments}

ACIP members (the membership roster for July 2015-June 2016 is available at http://www.cdc.gov/vaccines/acip/committee/members. html); ACIP Meningococcal Vaccine Work Group.

\begin{abstract}
${ }^{1}$ Division of Bacterial Diseases, National Center for Immunization and Respiratory Diseases, CDC; ${ }^{2}$ Advisory Committee on Immunization Practices Meningococcal Vaccines Work Group; Steven and Alexandra Cohen Children's Medical Center of New York, New Hyde Park, New York; and Hofstra Northwell School of Medicine, Hempstead, New York; ${ }^{3}$ Division of Viral Diseases, National Center for Immunization and Respiratory Diseases, CDC.
\end{abstract}

Corresponding author: Jessica R. MacNeil, jmacneil@cdc.gov.

\section{References}

1. Cohn AC, MacNeil JR, Clark TA, et al. Prevention and control of meningococcal disease: recommendations of the Advisory Committee on Immunization Practices (ACIP). MMWR Recomm Rep 2013 (No. RR-2).

2. Lujan-Zilbermann J, Warshaw MG, Williams PL, et al. Immunogenicity and safety of 1 vs 2 doses of quadrivalent meningococcal conjugate vaccine in youth infected with human immunodeficiency virus. J Pediatr 2012;161:676-81.e2.

3. Siberry GK, Warshaw MG, Williams PL, et al. Safety and immunogenicity of quadrivalent meningococcal conjugate vaccine in 2 - to 10 -year-old human immunodeficiency virus-infected children. Pediatr Infect Dis J 2012;31:47-52. http://dx.doi.org/10.1097/INF.0b013e318236c67b

4. Siberry GK, Williams PL, Lujan-Zilbermann J, et al. Phase I/II, openlabel trial of safety and immunogenicity of meningococcal (groups A, $\mathrm{C}, \mathrm{Y}$, and W-135) polysaccharide diphtheria toxoid conjugate vaccine in human immunodeficiency virus-infected adolescents. Pediatr Infect Dis J 2010;29:391-6. http://dx.doi.org/10.1097/INF.0b013e3181c38f3b

5. Schuchat A, Hilger T, Zell E, et al. Active bacterial core surveillance of the emerging infections program network. Emerg Infect Dis 2001;7:92-9. http://dx.doi.org/10.3201/eid0701.010114

6. Cohen C, Singh E, Wu HM, et al. Increased incidence of meningococcal disease in HIV-infected individuals associated with higher case-fatality ratios in South Africa. AIDS 2010;24:1351-60. http://dx.doi. org/10.1097/QAD.0b013e32833a2520

7. MillerL,ArakakiL, RamautarA, etal.Elevated risk for invasivemeningococcal disease among persons with HIV. Ann Intern Med 2014;160:30-7. http://dx.doi.org/10.7326/0003-4819-160-1-201401070-00731

8. Simmons RD, Kirwan P, Beebeejaun K, et al. Risk of invasive meningococcal disease in children and adults with HIV in England: a population-based cohort study. BMC Med 2015;13:297. http://dx.doi. org/10.1186/s12916-015-0538-6

9. Harris CM, Wu HM, Li J, et al. Meningococcal disease in patients with HIV infection-a review of cases reported through active surveillance in the United States, 2000-2008. Open Forum Infect Dis 2016. Epub October 24, 2016. http://ofid.oxfordjournals.org/content/ early/2016/10/24/ofid.ofw226.full.pdf+html

10. MacNeil JR, Rubin L, McNamara L, Briere EC, Clark TA, Cohn AC. Use of MenACWY-CRM vaccine in children aged 2 through 23 months at increased risk for meningococcal disease: recommendations of the Advisory Committee on Immunization Practices, 2013. MMWR Morb Mortal Wkly Rep 2014;63:527-30.

11. CDC. Licensure of a meningococcal conjugate vaccine for children aged 2 through 10 years and updated booster dose guidance for adolescents and other persons at increased risk for meningococcal disease-Advisory Committee on Immunization Practices (ACIP), 2011. MMWR Morb Mortal Wkly Rep 2011;60:1018-9. 
12. CDC. Recommendation of the Advisory Committee on Immunization Practices (ACIP) for use of quadrivalent meningococcal conjugate vaccine (MenACWY-D) among children aged 9 through 23 months at increased risk for invasive meningococcal disease. MMWR Morb Mortal Wkly Rep 2011;60:1391-2.

13. Food and Drug Administration. Menveo U.S. package insert. Silver Spring, MD: US Department of Health and Human Services, Food and Drug Administration; 2013. http://www.fda.gov/downloads/ BiologicsBloodVaccines/Vaccines/ApprovedProducts/UCM201349.pdf

14. Klein NP, Reisinger KS, Johnston W, et al. Safety and immunogenicity of a novel quadrivalent meningococcal CRM-conjugate vaccine given concomitantly with routine vaccinations in infants. Pediatr Infect Dis J 2012;31:64-71. http://dx.doi.org/10.1097/INF.0b013e31823dce5c
15. Nolan TM, Nissen MD, Naz A, et al. Immunogenicity and safety of a CRM-conjugated meningococcal ACWY vaccine administered concomitantly with routine vaccines starting at 2 months of age. Hum Vaccin Immunother 2014;10:280-9. http://dx.doi.org/10.4161/hv.27051

16. Food and Drug Administration. Menactra U.S. package insert. Silver Spring, MD: US Department of Health and Human Services, Food and Drug Administration; 2016. http://www.fda.gov/downloads/ BiologicBloodVaccines/Vaccines/ApprovedProducts/UCM131170.pdf 